\section{Low-cost, multimodal, portable screening system for early detection of oral cancer}

\author{
Mohammed Rahman, ${ }^{\text {a,b }}$ Pankaj Chaturvedi, ${ }^{b}$ \\ Ann M. Gillenwater, ${ }^{c}$ and Rebecca Richards-Kortum ${ }^{\mathrm{a}, *}$ \\ ${ }^{a}$ Rice University, Department of Bioengineering, 6100 Main \\ Street, Houston, Texas 77005 \\ bTata Memorial Hospital,Department of Surgery, Parel, \\ Mumbai 400012 \\ ${ }^{\mathrm{c}}$ M.D. Anderson Cancer Center, Head and Neck Surgery, \\ 1515 Holcombe Boulevard, Houston, Texas 77030
}

\begin{abstract}
Oral cancer is an important global health problem. There is an urgent need for improved methods to detect oral cancer and its precursors, because early detection is the best way to reduce oral cancer mortality and morbidity. In this work, we describe simple modifications to a surgical headlight system that enables direct visualization and digital image acquisition from oral tissue in multiple imaging modalities including fluorescence, white-light reflectance, and orthogonal polarization reflectance. Images obtained with the system in-vivo demonstrate that it is an attractive technology to explore for oral cancer screening in low-resource environments where clinical expertise is often unavailable. $\odot 2008$ Society of Photo-Optical Instrumentation Engineers. [DOI: 10.1117/1.2907455]
\end{abstract}

Keywords: oral cancer; surgical headlight; image acquisition; fluorscence imaging; white-light reflectance; orthogonal polarization reflectance.

Paper 07249LRR received Jul. 10, 2007; revised manuscript received Dec. 6, 2007; accepted for publication Dec. 27, 2007; published online Jun. 9, 2008.

Oral cancer is a major global health problem that disproportionately impacts patients in developing countries. According to the World Health Organization (WHO), the worldwide annual incidence for oral cancer exceeds 267,000 new cases with an estimated 128,000 deaths, nearly two-thirds of which occur in developing countries. ${ }^{1}$ Overall survival rates for oral cancer in developing countries are low, and there has been little improvement over the past three decades. In the vast majority of cases today, oral cancer is diagnosed at an advanced stage, when it requires more aggressive treatment and results in poor survival and increased morbidity. However, one large screening trial conducted in southern India demonstrated that early detection of oral cancer in high-risk populations can reduce mortality. ${ }^{2}$ Thus, new techniques that can aid diagnosis of changes associated with oral cancer at an early stage and can be implemented in low-resource settings would be an important contribution to global efforts to reduce oral cancer mortality and morbidity.

The standard method for screening and detection of oral neoplasia is visual inspection of oral cavity under white light. Unfortunately, the clinical appearance of oral cancers at an

*Tel: 713-348-5869; Fax: 713-348-5877; E-mail: rkortum@rice.edu early stage can be difficult to differentiate from nonspecific inflammation and irritation under white-light examination. Fluorescence imaging has been shown to be an effective alternative method for screening and diagnosis of precancers in several organ sites including oral cavity, uterine cervix, lung, and skin. ${ }^{3}$ Several groups, including Betz et al., Onizawa et al., Paczona et al., and Sivstun et al. have shown that examining the oral cavity under a fluorescence excitation light source can overcome some of the detection limitations associated with standard white-light examination. ${ }^{4-7}$ Lane et al. recently proposed a simple hand-held device for direct visualization of tissue autofluorescence above $480 \mathrm{~nm}$ using a metal halide mercury lamp with excitation wavelengths between 360 and $460 \mathrm{~nm}^{3}$ The device is currently approved for medical use by the Food and Drug Administration (FDA) in the United States.

Although previous fluorescence imaging devices have shown high sensitivity and specificity for detecting abnormalities in the oral cavity, their use has been mainly limited to medical facilities in developed countries. They are a less practical solution for mass screening of high-risk populations in low-resource settings, as the cost of these devices is relatively high, their portability is limited, and they require a stable, high-voltage power supply. Furthermore, it is difficult to use these devices to perform traditional white-light examination, which may prevent clinicians from obtaining clinical impressions they are accustomed to observing. In this work, we present a simple yet robust optical system that can be used for both white-light reflectance examination and fluorescence imaging. The head-mounted device presented here can be used for direct visualization by the health care provider, as well as for digital acquisition of images of the oral cavity. This inexpensive and portable device utilizes light-emitting diodes (LEDs) as its illumination source and can be powered by a compact lithium-ion battery. This approach not only makes the device low maintenance and affordable, but also makes it usable in low-resource settings where a stable source of electrical power may not be available. Altogether, the features of the optical device make it easily translatable to developing countries for screening oral cancer.

A schematic diagram of the optical device, named the portable screening system (PS2 Oral), is shown in Fig. 1. The complete system weighs only three pounds and consists of a commercially available surgical headlight system with loupes, light emitting diodes, a head-mounted charge-coupled device (CCD) camera, and a lithium-ion battery. The headlight system with binocular loupes was purchased from Heine USA Limited (Dover, New Hampshire). The $2.5 \times$ magnification binocular loupes provided a field of view (FOV) of $55 \mathrm{~mm}$ at a working distance (WD) of $250 \mathrm{~mm}$, with a depth of field (DOF) of $55 \mathrm{~mm}$. The spatial resolution of the system was tested with a USAF resolution target and up to four line pairs per millimeter could be resolved. The headlight system was modified to provide excitation light for fluorescence imaging with a blue LED (Luxeon Royal Blue-K2, Brantford, Ontario). The 750-mW rated LED provides a peak irradiance of $15 \mathrm{~mW} / \mathrm{cm}^{2}$ at the center of the measurement site at a peak wavelength of $455 \mathrm{~nm}$. An additional LED light source was incorporated to the system for white-light illumination. A re- 


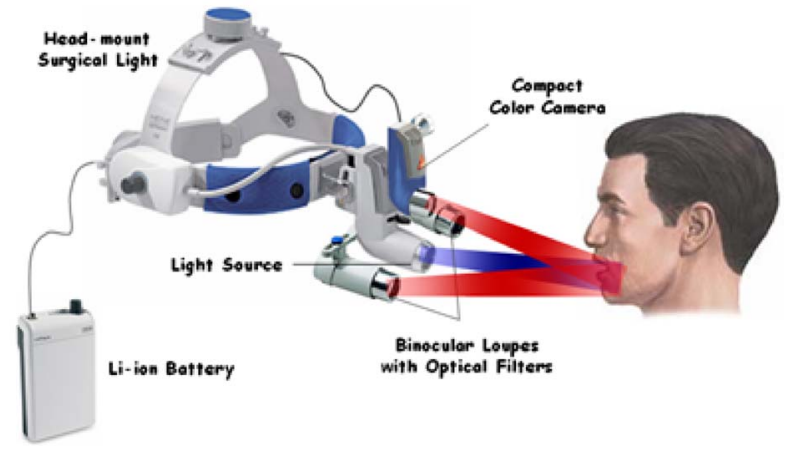

Fig. 1 A schematic diagram of the multimodal portable screening system for oral cancer (PS2 Oral).

chargeable lithium-ion battery provided with the headlight system from Heine USA was used to power both light sources and can be used continuously for four hours. To record digital images, a compact, head-mounted color CCD camera (Prosilica EC1380C, Burnaby, British Columbia) was integrated into the system. The camera can be powered and controlled by a laptop through an IEEE 1394 Firewire port. Images of objects on the camera were made parfocal with binocular loupes using a focus adjustable C-mount lens.

To examine and record tissue images in different modalities including fluorescence, standard white-light, and orthogonal polarized white-light modes, appropriate optical components were introduced in the optical light path. For fluorescence illumination, a 447/60-nm excitation filter (Semrock FF02-447/60) was placed in front of the blue LED to prevent any bleed-through above $480 \mathrm{~nm}$. The emitted fluorescence could be observed through the binocular loupes, which had single 480-nm long-pass emission filters (Omega Optical 480ALP) attached in front of each loupe to block fluorescence excitation light. Similarly, for orthogonal polarized light reflectance imaging, a linear polarizer was placed in front of the white LED light; an additional linear polarizer oriented at $90 \mathrm{deg}$ relative to the first was placed in the detection light path. This simultaneously blocks specular reflection and allow observation of lesions and deeper tissue structures such as microvasculature, which may not exhibit high contrast under standard white-light illumination. ${ }^{8}$ The longpass filter and polarizer in the detection path of the camera were placed using a custom designed filter holder. This holder contains three positions to allow different optical components to be easily interchanged during patient examinations in different imaging modalities. The total cost of the PS2 system as assembled here was approximately $\$ 4500$, excluding the cost of the PC. The system cost can be reduced to $\$ 1200$ by replacing the scientific-grade camera with a low-cost camera, which is sensitive enough to detect autofluorescence. We estimate that the final cost could be drastically reduced to less than $\$ 100$ by eliminating the camera altogether, but this would eliminate the capability for recording imaging data for clinical or research purposes.

To avoid interference from ambient light, all images including patient measurements were acquired in a dark room. Image acquisition was controlled using graphical user interface software developed in LabView. The system was used to obtain images of the oral cavity from normal volunteers and from patients suspected to have oral cancer at the University of Texas M.D. Anderson Cancer Center. Following image acquisition in consenting patients, clinically suspicious areas of tissue were biopsied and submitted for histopathologic diagnosis. In addition, the system was used to image the oral cavity of consenting patients at the Tata Memorial Hospital in Mumbai, India.

Figure 2 shows images of the floor of the mouth of a normal volunteer obtained using different imaging modalities with the portable screening system. Although the bright fluorescence signal from the teeth partially saturates the image, a green autofluorescence signal from tissue is clearly visible in Fig. 2(a). Similarly, in the standard white-light image, strong specular reflection in some regions partially saturates the image and hinders observation of underlying tissue structures. In comparison, in the orthogonal-polarized image, the specular reflection is removed, allowing good visualization of the subepithelial vasculature, as shown in Fig. 2(c).

Figure 3 shows images of the oral cavity from three patients obtained using the portable screening system. The top row shows images from the floor of the mouth of patient $\mathrm{A}$; the arrow in the fluorescence image indicates an area with strong loss of fluorescence; in contrast, the standard whitelight image and the orthogonal polarized white-light images of the same area showed no obvious clinical abnormalities. Both white-light images show the proposed region of tissue to be resected drawn by the surgeon based on clinical judgment. The histopathology report confirmed an area with dysplasia within this region.

In addition, Fig. 3 shows images from two patients with clinically apparent lesions. Although histopathology was not available in these patients, regions of loss of fluorescence correlate well with clinically suspicious areas. Images are shown from patients $\mathrm{B}$ and $\mathrm{C}$ with leukoplakia in the left buccal mucosa. In both cases, there is a strong loss of fluorescence associated with the area of leukoplakia. Images from patient B show a region of increased redness in the orthogonal polarized image just beneath the area of leukoplakia (arrows); this also corresponds to a region of decreased fluorescence. Images from the contralateral normal buccal mucosa in patient $\mathrm{C}$ show homogeneous fluorescence across the tissue.

The potential of the PS2 system to help differentiate between normal and dysplastic oral tissue is demonstrated in Figs. 2 and 3. In contrast to the images from the normal volunteer, the autofluorescence signal from dysplastic tissue in patient A shows a characteristic loss of autofluorescence. Similarly, in patients B and C, mucosal areas with lesions clinically suspicious for dysplasia also show characteristic loss of autofluorescence. This is consistent with previous research using more costly scientific-grade instruments; this loss of autofluorescence is attributed to a decrease in stromal collagen and elastin fluorescence that underlies the dysplastic epithelial tissue. ${ }^{9}$ Furthermore, the use of orthogonal polarization imaging reduces interfering specular reflection and enhances contrast associated with subsurface vasculature, which is frequently increased in neoplastic lesions. ${ }^{8}$

In summary, we have developed a low-cost, portable imaging device that has the potential to be used to improve oral cancer screening. The device incorporates LED illumination, and can be used in both white-light and fluorescence imaging 


\section{JBO LETTERS}
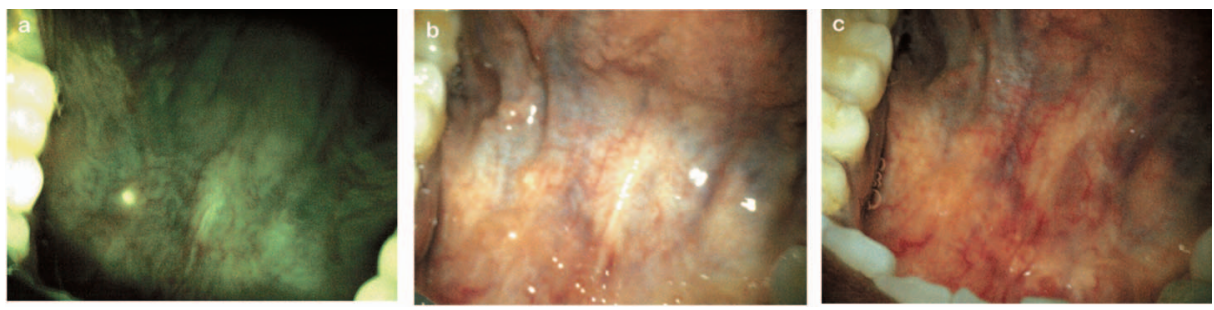

Fig. 2 Digital images of the floor of the mouth from a normal volunteer using the portable screening system: (a) fluorescence (b) standard white-light reflectance, and (c) orthogonal polarized white-light reflectance images.

modes. It is battery powered and can acquire digital images of tissue with an integrated CCD camera. These stored images can be used in long-term clinical surveillance, for telemedicine review by clinical experts, and potentially for incorporation of automated image analysis algorithms. Overall, these features, coupled with the low cost of the system, make it an attractive option to explore for oral cancer screening in lowresource settings. Further clinical trials in a controlled, prospective setting are required to evaluate the diagnostic performance of the device and to determine the degree of clinical expertise required to use it effectively. Athough the device is designed for screening for oral cancer, it can be translated to other organ sites. Furthermore, as new understandings in optical properties of normal and diseased tissue are conceived

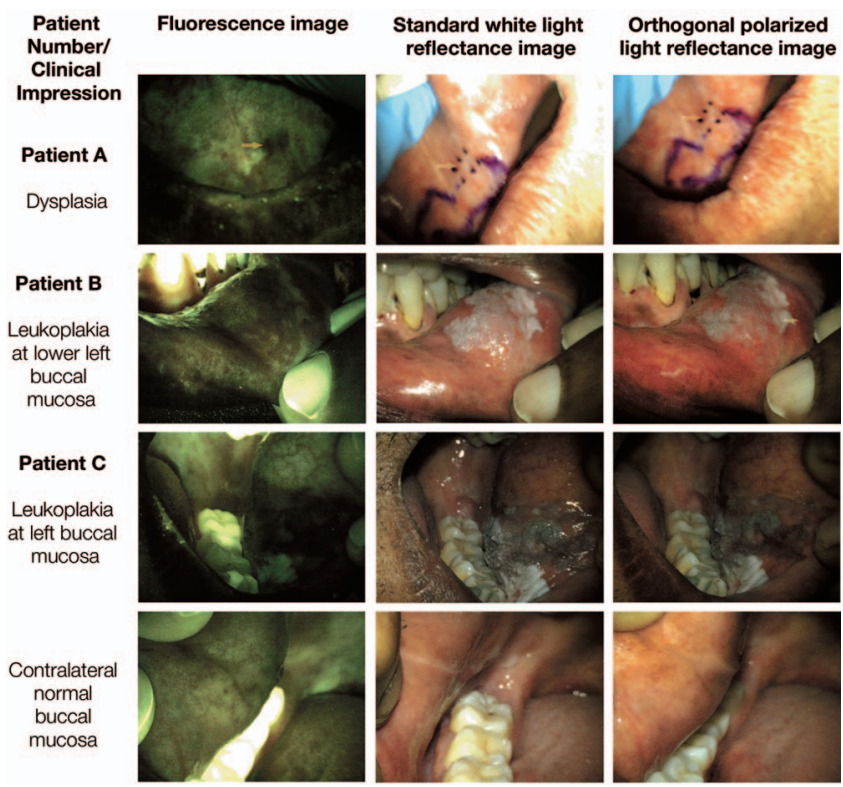

Fig. 3 Images of the oral cavity from three patients obtained with the portable screening system: (left column) fluorescence images, (middle column) standard white-light reflectance images, and (right column) orthogonal polarized white-light reflectance images. and advances in LED technologies are made, they can be easily incorporated into the PS2 system to improve the device.

\section{Acknowledgments}

The authors acknowledge grant FIC R03 TW007910. They would also like to thank Dr. Anil D'Cruz from Department of Surgery and Dr. Surendra Shastri from Department of Preventive Oncology at Tata Memorial Hospital, Mumbai, India for their support in the work.

\section{References}

1. J. Ferlay, F. Bray, P. Pisani, and D. M. Parkin, "GLOBOCAN 2002: cancer incidence, mortality and prevalence worldwide," IARC CancerBase No. 5, version 2.0 ed., IARC Press, Lyon (2004).

2. R. Sankaranarayanan, K. Ramadas, G. Thomas, R. Muwonge, S. Thara, B. Mathew, and B. Rajan, "Effect of screening on oral cancer mortality in Kerala, India: a cluster-randomised controlled trial Cancer survival in developing countries," Lancet 365, 1927-1933 (2005).

3. P. Lane, T. Gilhuly, P. Whitehead, H. Zeng, C. Poh, S. Ng, P. Williams, L. Zhang, M. Rosin, and C. MacAulay, "Simple device for the direct visualization of oral-cavity tissue fluorescence," J. Biomed. Opt. 11(2), 24006 (2006).

4. C. S. Betz, M. Mehlmann, K. Rick, H. Stepp, G. Grevers, R. Baumgartner, and A. Leunig, "Autofluorescence imaging and spectroscopy of normal and malignant mucosa in patients with head and neck cancer," Lasers Surg. Med. 25, 323-334 (1999).

5. R. Paczona, S. Temam, F. Janot, P. Marandas, and B. Luboinski, "Autofluorescence videoendoscopy for photodiagnosis of head and neck squamous cell carcinoma," Eur. Arch. Otorhinolaryngol. 260, 544-548 (2003).

6. K. Onizawa, H. Saginoya, Y. Furuya, H. Yoshida, and H. Fukuda, "Usefulness of fluorescence photography for diagnosis of oral cancer," Int. J. Oral Maxillofac Surg. 28, 206-210 (1999).

7. E. Svistun, R. Alizadeh-Naderi, A. El-Naggar, R. Jacob, A. Gillenwater, and R. Richards-Kortum, "Vision enhancement system for detection of oral cavity neoplasia based on autofluorescence," Head Neck 26, 205-215 (2004).

8. S. L. Jacques, J. C. Ramella-Roman, and K. Lee, "Imaging skin pathology with polarized light," J. Biomed. Opt. 7(3), 329-340 (2002).

9. I. Pavlova, K. Sokolov, R. Drezek, A. Malpica, M. Follen, and R. Richards-Kortum, "Microanatomical and biochemical origins of normal and precancerous cervical autofluorescence using laser-scanning fluorescence confocal microscopy," Photochem. Photobiol. 77, 550555 (2003). 